\title{
RISK ELEMENTS AND DRUG UTILIZATION IN STROKE PATIENTS
}

\author{
SPURTHI T.*, GOWTHAMI B., KHYATHI D., VINOD G. \\ Department of Pharmacy Practice, Creative Educational Society's College of Pharmacy, N. H.7, Chinnatekur, Kurnool, Andhra Pradesh \\ Email: t.spurthireddy@gmail.com
}

Received: 15 May 2016 Revised and Accepted: 12 Aug 2016

\begin{abstract}
Objective: Cerebrovascular accident (CVA), formally called as stroke is the second leading cause of death worldwide. Recent studies in different parts of India also documented that the prevalence of stroke varies from 40 to 270 per 100,000 populations. Identifying the risk factors of the disease helps to improve the effectiveness of the therapy. Our present study aims in accessing the risk aspects and utilization of drugs in CVA patients.

Methods: A prospective Interventional study was carried out on stroke patients in Medicine Department, Viswabarathi Hospital, Kurnool, AP from January to September 2015. Required demographic and laboratory data, along with general neurological evaluation and brain CT was analyzed.

Results: A total of 100 patients was analyzed. Among them, the incidence of ischemic stroke (96\%) was greater compared to hemorrhagic stroke (4\%). Prevalence of disease was higher in males (69\%) than females (31\%) with notable occurrence in the age group of 61-70 (26\%). Higher percentage of patients were found to have>3 risk factors (34\%), including hypertension (68\%), age (58\%), diabetes (39\%), smoking (22\%), alcoholism (21\%), CAD (10\%) and percentage with no risk element accounts7\%. The prescribing pattern includes antihypertensives (16.25\%), Gastrointestinal agents (12.2\%), anti-platelets (9.16\%), hyperlipidemic (8.4\%), Nootropics (7.97\%) followed by other classes and nitrates $(0.62 \%)$ occupies the last place in prescribing.
\end{abstract}

Conclusion: The present study emphasizes on the need to identify risk factors and providing awareness among the patients by a pharmacist in minimizing the disease burden. It also points the rationality in prescribing of drugs based on therapeutic guidelines.

Keywords: Stroke, Risk factors, Prescribing patterns, Ischemia

(C) 2016 The Authors. Published by Innovare Academic Sciences Pvt Ltd. This is an open access article under the CC BY license (http://creativecommons. org/licenses/by/4. 0/) DOI: http://dx.doi.org/10.22159/ijpps.2016v8i10.12870

Disease affecting the supply of blood to the brain is referred as stroke or Cerebrovascular diseases or apoplexy. WHO has defined a stroke as "rapidly developing clinical signs of focal or global disturbance of cerebral function, lasting for more than twenty-four hours or leading to death, with no apparent cause other than the vascular origin. Epidemiological studies state that stroke is the third leading cause of death worldwide along with coronary artery disease. A survey in different parts of India also shows that it differs from region to region, ranging from 40 to 270 per 100,000 populations [1].

Based on clinical syndrome, stroke can be divided mainly as Ischemic, causing an acute occlusion of blood flow to the brain due to a thrombus of cerebral origin or from another part of circulation and hemorrhage, caused by a subarachnoid hemorrhage in brain tissues or intra-cerebral hemorrhage, i.e., between meningeal spaces. Among both $50-80 \%$ of stroke is contributed by ischemic pathophysiology. The prevalence of the disease is very much closely related to its risk factors, categorized as a) fixed risk factors (Age, Gender, Race, previous vascular event (MI, stroke, PVD), Hereditary (high Fibrinogen)) and b) Modifiable risk factors (Blood pressure, Cigarette, smoking, hyperlipidemia, Heart disease (Atrial fibrillation, Congestive Cardiac Failure (CCF), infective endocarditis), Diabetes mellitus, Excessive alcohol intake, estrogen-containing drugs (OC pills, HRT), Polycythemia) [2].

According to standard guidelines, early recognition by fast analysis of symptoms like the weakness of upper and lower limbs, face as well as speech and evidential diagnosis using validated tools like CT and MRI scan or PET and ultrasound help in saving lives with a minimal disability. Therapeutic options of Stroke include primary management with antiplatelet therapy along with aspirin, statin therapy and blood pressure management. Secondary management with carotid endarterectomy, carotid angioplasty, warfarin and heparin is useful. Calcium antagonists like oral nimodipine is useful in hemorrhagic stroke [3].
Even though many researchers worked on stroke-related risk factors, the reporting of its incidence in Indian population creates a helping tool for better patient care and best medical attention. Our present prospective study aims to study both fixed and modifiable risk factors, which help in providing awareness and development of preventive measures along with drug utilization study for setting appropriate interventions to identify the problems in prescribing practices and also in promoting rational use of drugs in the community.

This study was conducted in the Medicine department of Viswabarathi Hospital, which is 250 bedded tertiary care teaching hospital providing health care services. The research approach adopted in this study was a prospective study in the Medicine Department of a tertiary care teaching hospital, for a period of three months from January 2015 to September 2015.

This study includes hospital in patients treated for Stroke in Medicine department. A patient who meets the following criteria was enrolled where, the inclusion criteria were patients aged $\geq 18 \mathrm{y}$ of both genders and age group of 18-90 y. Exclusion criteria were patients with diseases like psychiatry, cancer, Pregnant and nursing mothers.

The cases which had found in Medicine department, details of the cases, including patient name, age, sex, past medical history and other relevant information was collected. The collected prescriptions were entered into the Microsoft Office Excel sheet according to their age, gender, therapeutic category, and prescription and was approved by the Institutional Human Ethics Committee and Informed consent from patients was taken.

A total of 100 prescriptions was analyzed. 69\% were male patients, and $31 \%$ were female mentioned in fig. 1 . Out of which ischemic stroke (96\%) is greater compared to hemorrhagic stroke (4\%) as cited in fig. 2. Maximum patients were seen in the age group of $61-70(26 \%)$ and the least were seen in the age group 20-30 (2\%) as in table 1 . 


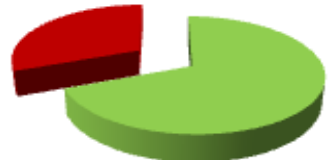

MALES (69\%)

Fig. 1: Gender distribution of CVA

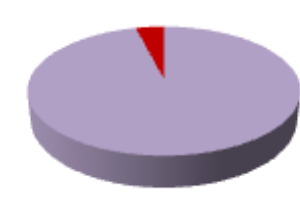

- ISCHEMIC (96\%) — HEMORRHAGIC (4\%)

Fig. 2: Incidence of stroke

Table 1: Demographic distribution of stroke in different age groups

\begin{tabular}{lll}
\hline Age group (in years) & Percentage distribution & Total N=100 \\
\hline $21-30$ & $2 \%$ & 2 \\
$31-40$ & $7 \%$ & 7 \\
$41-50$ & $20 \%$ & 20 \\
$51-60$ & $20 \%$ & 20 \\
$61-70$ & $26 \%$ & 19 \\
$71-80$ & $19 \%$ & 6 \\
$81-90$ & $6 \%$ & \\
\hline
\end{tabular}

Most of the CVA patients exhibited common symptoms like weakness, hemiparesis, and difficulty to talk. In the study population, highest percentage showed $>3$ risk factors (34\%) followed by 2 risk factors (27\%), $3(23 \%)$ and $1(9 \%)$ risk elements. Around $7 \%$ of patients also reported with no risk involved.

Hypertension was the most common risk factor $68(68 \%)$ followed by age $58(58 \%)$, diabetes mellitus 39 (39\%), smoking $22(22 \%)$, alcoholism 21 (21\%) and coronary artery disease 10 (10\%) mentioned in table 2 along with their percentages.

The patients are treated with a different class of drugs among which anti-platelets $88(91.16 \%)$ accounts the highest percentage and nitrates $6(0.62 \%)$ is the least prescribed. The remaining classes of drugs and their percentage of usage is mentioned in following table 3 .

Table 2: Different risk factors associated with stroke

\begin{tabular}{lll}
\hline Risk factors & Percentage (\%) & Total N=100 \\
\hline HTN & $68 \%$ & 68 \\
DM & $39 \%$ & 39 \\
AGE & $58 \%$ & 58 \\
ALCHOLIC & $21 \%$ & 21 \\
SMOKING & $22 \%$ & 22 \\
CAD & $10 \%$ & 10 \\
\hline
\end{tabular}

HTN=Hypertension; DM=Diabetes mellitus; $\mathrm{CAD}=$ coronary artery disease

Table 3: Drug utilization pattern in stroke patients

\begin{tabular}{ll}
\hline Class of drugs & No of drugs \\
\hline Antiplatelets & 88 \\
Antihypertensives & 157 \\
Gastrointestinal agents & 118 \\
Hypolipidemic agents & 82 \\
Antibiotics & 79 \\
Nootropics & 77 \\
Anti-Convulsants & 65 \\
Analgesics and antipyretics & 48 \\
Insulin & 25 \\
Anti-vertigo & 16 \\
Hypoglycemic agents & 14 \\
Nitrates & 6 \\
\hline
\end{tabular}

The stroke is considered as a emergency medical condition which is to be treated as early as possible. Other than ischemic and hemorrhagic stroke, they are other conditions like TIA (transient ischemic attack) and silent stroke. TIA also called as mini-stroke or warning stroke. It takes the definition that stoppage of blood flow or ischemia to a part of the brain for a small period of time. Whereas silent stroke is the condition which doesn't show any symptoms, but manifested by ischemic attacks. It is often undetectable and reversible. A silent stroke is also called as silent cerebral infarct. Apart from risk factors, a major complication in stroke patients is the risk of falls. It might implicate multiple factors even such as age, gender, hypertension, diabetes, alcohol and smokers [4]. Out of collecting prescriptions, we have seen a majority of patients in ischemic stroke and coverage of little percentage with respect to hemorrhagic stroke. This was in correlation to the study conducted by Indira Kumari N and Veera Raghavulu B by Andhra University, Visakhapatnam [5].

It was found that higher incidence of stroke was found in males than in females. This might be due to the increased stress levels in males and protective estrogen effects in females. This was with respect to the study conducted by Md. Abdul Muhit et al., in Journal of Applied Pharmaceutical Science [2].

Age wise distribution of stroke patients shown that peak incidence was seen in the age group of 61-70 y followed by 41-50 y and 51-60 $\mathrm{y}$ with equality in distribution. A similar study conducted by Adepu 
Ramesh on Assessment of drug-related problems in stroke patients admitted to a South Indian tertiary care teaching hospital shown a peak incidence of stroke in the age group of 60-69 y followed by the age group of 50-59 y [6].

We already knew that as the number of risk factors increases, the progression and complications of the disease increases. Even in the present study, we found that there were more patients who have involvement of multiple risk factors than single or no risk factors. Proper intervention is to be done assessing risk factors. And measures are to be taken in order to avoid progression of certain risk factors. Because improper control on stroke leads to disability [1].

The major risk factor in stroke patients in the present study was found to be hypertension followed by diabetes and age. And the least risk was seen with a past history of coronary artery disease. The reasons for the progression with these risk factors to the stroke might be due to change in capillary tone and advances glycation product formation. This was similar to the study conducted by Indira Kumari N and Veera Raghavulu B in International Journal of Research in Health Sciences [5].

Proper management reduces the incidence and progression of the disease. Drug utilization pattern provides the rationality in drug prescribing and analysis. It has shown that there was a higher incidence of utilization of anti-hypertensive drugs followed by gastrointestinal and anti-platelet agents with the last prescription of nitrates. But a similar study of drug utilization pattern in stroke patients conducted by Subash Vijaya Kumar et al., shown the highest usage of gastrointestinal agents followed by antibiotics and monitor with the least use of antiplatelet agents [7].

The study could not assess the complications and their therapy as, follow-up was not there. Finally, we conclude that proper risk factor management and following the guidelines in the treatment reduces the severity, thereby the prognostic factors will be good. Early identification of risk factors and pattern of therapy plays a crucial role in qualitative patient care.
The authors duly acknowledge the contribution and help patients. We also thank our principal and faculty members of Department of Pharmacy Practice, CES College of Pharmacy, Kurnool, A. P for their support and valuable guidance in this work. We wish to express our gratitude to the Medicine Department of Viswabarathi Hospital for their support and their cooperation in issuing the Medical reports.

\section{CONFLICTS OF INTERESTS}

\section{Declared none}

\section{REFERENCES}

1. Prakash Gurudevarahali Madegowda, Sanju R. Risk factors in patients with the ischemic stroke-A prospective study. Int Arch Integrated Med 2015;2:28-39.

2. Md Abdul Muhit. Cardiovascular disease prevalence and prescription patterns at a tertiary hospital in Bangladesh. J Appl Pharm Sci 2012;2:80-4.

3. Monaliza, Meena A, Achal S. Awareness of risk factors and warning symptoms of stroke in the general population. Nursing Midwifery Res J 2012;8:149-61.

4. Atzmon Tsur, Zvi Segal. Falls in stroke patients: risk factors and risk management. Isr Med Assoc J 2010;12:216-9.

5. Indira kumari N, Veera Raghavulu B. Risk factor assessment of stroke and its awareness among stroke survivors: a retrospective study. Int J Res Health Sci 2015;3:140-5.

6. Celin AT, Seuma J, Ramesh A. Assessment of drug-related problems in stroke patients admitted to a south indian teritiary care teaching hospital. Indian J Pharm Practice 2012;5:28-33.

7. Subhash Vijaya kumar, G Damodar, S Ravikanth, G Vijaya kumar. Drug utilization pattern of stroke patients in a tertiary care hospital. Int J Curr Pharm Clin Res 2012;2:3-7.

\section{How to cite this article}

- $\quad$ Spurthi T, Gowthami B, Khyathi D, Vinod G. Risk elements and drug utilization in stroke patients. Int J Pharm Pharm Sci 2016;8(10):290-292. 